\title{
230 PRECLINICAL EFFICACY OF CLEC-1 ANTAGONIST AS NOVEL MYELOID IMMUNE CHECKPOINT THERAPY FOR ONCOLOGY
}

${ }^{1}$ Vanessa Gauttier*, 'Marion Drouin, 'Sabrina Pengam, ${ }^{2}$ Javier Saenz, ${ }^{2}$ Bérangère Evrard, ${ }^{1}$ Stéphanie Neyton, ${ }^{1}$ caroline Mary, ${ }^{1}$ Géraldine Teppaz, ${ }^{1}$ Ariane Desselle, ${ }^{1}$ Virginie Thépénier, ${ }^{1}$ Emmanuelle Wilhelm, ${ }^{1}$ Nicolas Poirier, ${ }^{2}$ Elise Chiffoleau. ${ }^{1}$ OSE Immunotherapeutics, Nantes, France; ${ }^{2}$ CRTI - UMR1064, Nantes, France

Background C-type lectin receptors (CLRs) are powerful pattern recognition receptors shaping immune cell-mediated tissue damage by positively or negatively regulating myeloid cell functions and hence tumor elimination or evasion. We previously reported that the orphan CLR CLEC-1 expressed by dendritic cells (DCs) tempers T cell's responses in vivo by limiting antigen cross-presentation by cDC1. Furthermore, we observed that CLEC-1 is highly expressed by myeloid cells purified from human tumor microenvironment, in particular tumor-associated macrophages.

Methods Macrophages were generated from monocytes of healthy volunteers for phagocytosis assays. MC38 and Hepa 1.6 murine tumor cells were implanted in Clec1a $\mathrm{KO}$ or KI mice for immunotherapeutic treatment evaluation.

Results Using newly developed anti-human CLEC-1 monoclonal antibodies (mAbs), we found that antagonist anti-CLEC-1 mAbs with the capacity to block CLEC-1/CLEC-1Ligand interaction, as opposed to non-antagonist CLEC-1 mAbs, increase the phagocytosis of CLEC-1Ligand-positive human tumor cells by human macrophages, in particular when opsonized by tumor-associated antigen mAbs (Rituximab, Cetuximab, Trastuzumab) or with anti-CD47 mAb (Magrolimab). In-vivo, CLEC1 knock-out $(\mathrm{KO})$ mice $(\mathrm{n}=19)$ display significant prolonged survival in monotherapy as compared to wild-type littermates $(n=12)$ in an orthotopic hepatocellular carcinoma (HCC) model and anti-tumor memory responses was demonstrated by tumor rechallenge in cured mice. CLEC1 KO mice also illustrate significant eradication of MC38 colorectal tumors in combination with chemotherapy promoting CLEC-1Ligand expression by tumor cells ( $n=16$ with Gemcitabine or $n=11$ with Cyclophosphamide). HCC tumor microenvironment analysis after 2 weeks of tumor implantation shows significantly higher number of $\mathrm{CD} 8+$ and memory $\mathrm{CD} 8+\mathrm{T}$ cells with reduced PD1 expression in CLEC1 KO animals ( $n=16$ versus $\mathrm{n}=12$ for KO vs WT mice respectively). Finally, we recently generated human CLEC-1 knock-in mice expressing the extracellular human CLEC1 domain fused to the intracellular mouse CLEC1 tail and confirmed preclinical efficacy in vivo with anti-human CLEC1 antagonist $\mathrm{mAb}$ in monotherapy in the orthotopic HCC model.

Conclusions These data illustrate that CLEC-1 inhibition represents a novel therapeutic target for immuno-oncology modifying $\mathrm{T}$ cell immune responses and tumor cell phagocytosis by macrophages.

http://dx.doi.org/10.1136/jitc-2021-SITC2021.230 